\title{
The influence of CDN sinking on metro transmission network
}

\author{
$Y$. $\mathrm{Hu}^{*}$, and $P$. Peng \\ China Mobile Communications Group Zhejiang Co., Ltd. Hangzhou branch, Hangzhou, China, \\ 310015
}

\begin{abstract}
With the rise of $\mathrm{VR} / \mathrm{AR}$ and $4 \mathrm{~K} / 8 \mathrm{~K}$ video services, users have higher requirements for personal experience. Promotion of user experience is an important factor to attract end-users. Compared with traditional website access, the deployment of $\mathrm{CDN}$ has advantages of improving access speed, solving the problem of high traffic and large concurrency, improving the reliability of data sources. CDN's basic characteristics can be summarized as: cache contents in different locations, and load requests of users to the most appropriate cache server through load balancing technology. The traditional networking mode deployed CDN on the core layer leads to contents away from the end users. This means the probability of occurrence of congestion increased, and the end-to-end delay enhanced. Therefore, CDN sink to the convergence layer and even edge layer can be considered to solve problems such as web browsing slow, video buffering slow and so on. The sinking of CDN has a profound impact on the network structure, basic resources and network intellectualization. Large capacity PTN/OTN equipment should be deployed on each layer. The sinking of CDN leads to the flattening of the network. The basic resources of metro transport network such as computer room space, air conditioning, power supply must ensure the normal operation of the sinking CDN equipments and transmission equipments. SDN/NFV technology should be introduced to transmission network in the future.
\end{abstract}

\section{Introduction}

With the "Internet plus" national strategic level to promote, $\mathrm{CDN}$ is playing a more and more important "Internet industry accelerator" role, to promote the rapid development of the Internet industry. With the rise of $\mathrm{VR} / \mathrm{AR}$ and $4 \mathrm{~K} / 8 \mathrm{~K}$ video services, users have higher requirements for personal experience [1]. Promotion of user experience is an important factor to attract end-users. With network latency reduced by 100 milliseconds, site visits will increase by $2.25 \%$, and revenue will rise by $1 \%$. The traditional networking mode deployed $\mathrm{CDN}$ on the core layer can not been able to meet the latency request. Therefore, CDN sink to the convergence layer and even edge layer can be considered to solve problems such as web browsing slow, video buffering slow and so on.

\footnotetext{
*Corresponding author: huyuzju@hotmail.com
} 


\section{The demand of the present network on CDN sinking}

CDN consists of source stations, cache servers, intelligent DNSs, and clients. By reducing the number of network devices between users and contents, and shortening the distance between the content source and users, $\mathrm{CDN}$ enhance the network performance [2]. Its basic characteristics can be summarized as: cache contents in different locations, and load requests of users to the most appropriate cache server through load balancing technology. Compared with traditional website access, the deployment of CDN has the following advantages:

(1) Improving access speed. CDN adds buffer layer between users and servers, and guides users' access requests to the optimal cache nodes instead of server source sites, thereby accelerating access speed.

(2) Solving the problem of high traffic and large concurrency. Users can get cached data directly from $\mathrm{CDN}$ after deploying $\mathrm{CDN}$. Concurrency rate will be reduced because data will not be obtained from the source site.

(3) Improving the reliability of data sources. When a source site fails, the user can obtain data from the CDN cache.

At present, the main application scenarios of CDN include improving the speed of static web browsing, improving the speed of large file download, dynamic acceleration of live websites, mobile APP dynamic acceleration and so on. With the extensive application of 5G, $4 \mathrm{~K} / 8 \mathrm{~K}$ and $\mathrm{VR} / \mathrm{AR}$ technologies, the requirements of large bandwidth and ultra low delay will be the key to the deployment of $\mathrm{CDN}$ and the key to the construction of metropolitan area network [3]. CDN deployed at the core layer leads to contents away from the end users. This means the probability of occurrence of congestion increased, and the end-to-end delay enhanced. The impact on network latency and download speed of deployment of CDN are plotted in figure 1. Network delays increased and download speed decreased with the transmission distance increased.

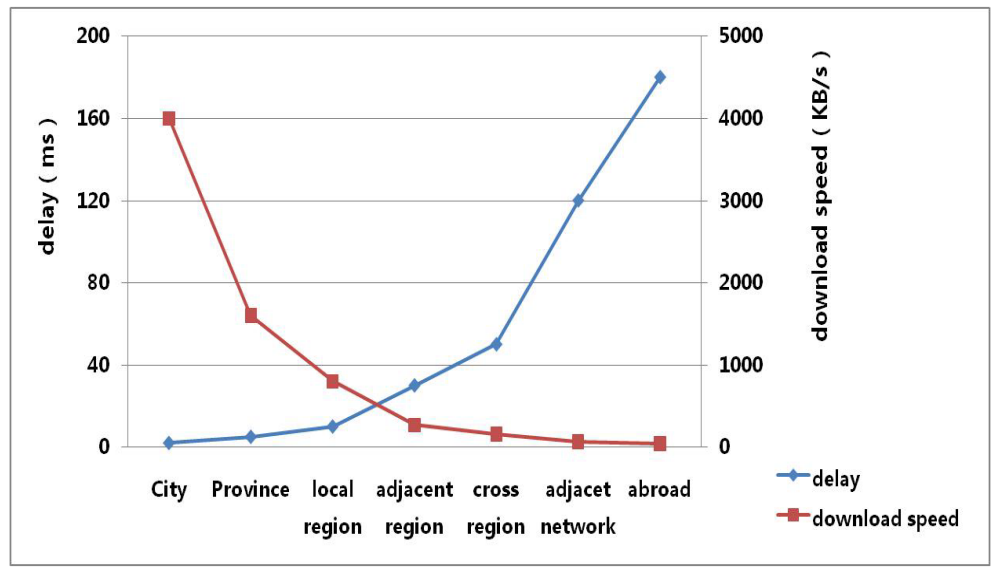

Fig. 1. Impact of CDN deployment locations on delays and download speeds.

Therefore, in order to enhance the personal experience, it is necessary to reduce the network jump node by deploying the CDN close to the user side. This makes network structure achieving flat. The personal experience of users is improved. As shown in figure 2, three layer architectures instead of two layer architectures will be applied in CDN system structure. CDN will gradually sink to the metro convergence layer from core layer. OLT nodes can be deployed in some hot spots. The regional hot resource storage can be deployed by means of independent CDN devices or OLT integrated Mini CDN.

In the future, the transmission network will introduce SDN/NFV technology to realize the network cloud and resource pool. The deployment and traffic scheduling of CDN can be 
realized through the control plane of the whole network. Therefore, the deployment of CDN can be determined by the control plane network after SDN/NFV technology introduced to metro transmission network [4].

CDN located in the core layer

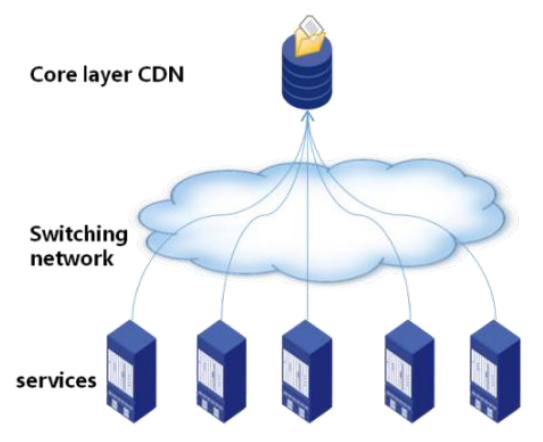

north-south traffic flow
CDN located in the convergence layer

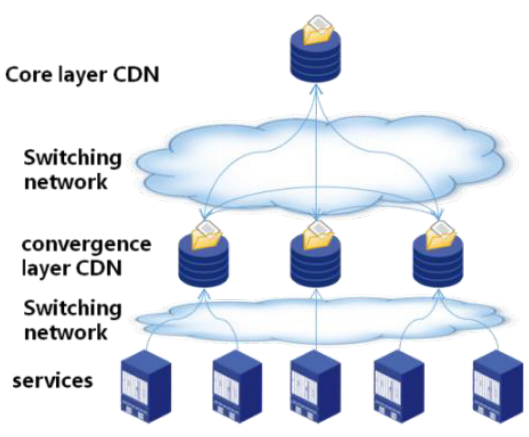

east-west traffic flow

Fig. 2. network architecture changes driven by CDN sinking.

\section{The influence of CDN sinking on metro transmission network}

The sinking of CDN has a profound impact on the network structure, basic resources and network intellectualization.

\subsection{Bandwidth requirement}

High-definition video, VR/AR and other large bandwidth, low latency services are carried on CDN. With the development of these large particle services and the sinking of CDN, which are loading these services, it will inevitably lead to a substantial increase in the flow of traffic on each layer of network. The transmission network capacity should be gradually improved to meet the bandwidth requirements after CDN sinking.

(1) $10 \mathrm{G}$ PON and 10G access devices should be deployed on access layer. In services intensive area, OLT sinking will be optimized, shorten the access distance, reduce the number of users brought by single OLT, improve the quality of broadband access, and achieve the upgrading of access loop from GE to 10GE.

(2)100G large capacity OTN/PTN equipment will be deployed on convergence layer. The corresponding ring capacity upgrades for the 100GE/200GE.

(3)The core layer will deploy 400GE OTN equipment, and upgrade to 400GE loop.

\subsection{Changing in network architecture}

The sinking of CDN leads to the flattening of the network. From the point of view of the CDN sinking, the power of the network tends to flattening from two aspects. On the one hand, lots of contents can be got directly from edge layer instead of caught from core layer after CDN deployed from the core layer to edge. East-west traffic flow increases and north-south traffic flow decreases. This makes the metro transmission network traffic flow getting more complicated than ever. Flatten network structure can simplify the traditional network hierarchy and network structure. On the other hand, the service loaded by CDN is 
very demanding for time delay, only flatten network can meet the requirement of low delay services.

Delay is mainly generated from optical fiber transmission and forwarding in equipment.

E2E delay is calculated as:

$\mathrm{E} 2 \mathrm{E}$ delay $=$ application delay at both ends + device delay $\times 2+$ fiber delay $\times 2$

Take high-definition video as an example, application delay of single end is $2 \mathrm{~ms}$, device delay is $60 \mathrm{us}$, optical fiber delay is $10 \mathrm{us} / \mathrm{km}$. If total delay cannot exceed $10 \mathrm{~ms}$, the fiber length should not exceed $328 \mathrm{~km}$. Metro transmission network should be meshed in the convergence layer. This can provide multi transmission paths, reduce cross node hops, reduce transmission delay, and improve the network transmission function. In addition, all-optical cross can be applicated in metro transmission network to reduce cross processing delay, greatly increase cross direction and improve cross processing capability.

\subsection{Basic resources requirement}

The basic resources of metro transport network such as computer room space, air conditioning, power supply must ensure the normal operation of the sinking CDN equipments and transmission equipments. In order to ensure the normal work of the sinking CDN equipment and transmission equipment, the space of the data center must be equipped with the ability to accommodate new equipment. The power supply must be able to meet the new load requirements and provide the required length of backup power supply. The effect of air conditioning and refrigeration should also meet the needs of new equipment, which will not cause the room temperature to rise in the room. The route of access to the data center should satisfy the laying of the optical cable to the data center, so as to ensure that the route resources of the data center can guarantee the demand of the network.

\subsection{Introduction of SDN/NFV Technology}

In the future, SDN/NFV and other technologies can be introduced to build an intelligent network. On the one hand, traffic load balancing is used to reduce the probability of network congestion and reduce the rate of packet loss by traffic monitoring. On the other hand, when network congestion occurs, it can quickly and conveniently calculate the scheduling path, dredge the network in time, and deal with the situation of traffic burst. probability of occurrence of network congestion and packet loss rate will greatly decreased by network traffic monitoring system after SDN/NFV technology introduced to metro transmission network[5].

\section{Conclusions}

CDN equipments will gradually sink to convergence layer. This makes the content closer to the user side, and user experience will improved. Capacity of metro transmission network should be promoted, network structure should be flattened, basic resources should be guaranteed and SDN/NFV technology should be introduced to transmission network, respectively.

\section{References}

1. M Ponder, G Papagiannakis, T Molet, N Magnenatthalmann, D ThalmannVHD++ Development Framework: Towards Extendible, Component Based VR/AR Simulation Engine Featuring Advanced Virtual Character Technologies, Computer Graphics 
International, 2003, 2003:96-104

2. G Peng, Research Proficiency Exam Report, 2004:1-6

3. D Xu,SS Kulkarni,C Rosenberg,HK Chai, Analysis of a CDN---P2P hybrid architecture for cost-effective streaming media distribution, Multimedia Systems,2006,11 (4) : 383-399

4. N Feamster, J Rexford, E Zegura, The road to SDN:an intellectual history of programmable networks, Acm Sigcomm Computer Communication Review, 2014, 44 (2): $87-98$

5. M Wichtlhuber, R Reinecke, D Hausheer An SDN-Based CDN/ISP Collaboration Architecture for Managing High-Volume Flows, Network \& Service Management IEEE Transactions on, 2015, 12(1):48-60 